\title{
Enhanced data assimilation of 4D LPT with physics informed neural networks
}

\author{
Jengmin Han ${ }^{1 *}$, Dong Kim ${ }^{1}$, Hyungmin Shin $^{1}$, Kyung Chun Kim ${ }^{1}$ \\ ${ }^{1}$ Pusan National University, School of Mechanical Engineering, Busan, South Korea \\ *hoon8674@pusan.ac.kr
}

\begin{abstract}
According to recent trend of explosive growth of computation power and accumulated data, demand for the deep learning application in various research fields is increasing. As following this trend, remarkable achievements are presented in the experimental fluid mechanics field. One of the most outstanding research is Physics Informed Neural Networks (PINN) Raissi et al. (2020). Physical knowledge, which has been accumulated by humans, is imposed on the neural networks. PINN was used the automatic differentiation for implementing the governing equations as a physical constraint. By utilizing this concept, physical constraints make neural networks finding physical meaning of phenomena instead of simply fitting to the label data.
\end{abstract}

Kim et al. (2021) conducted velocity field measurements of flow past the side mirror model by 4D Lagrangian PTV (Particle Tracking Velocimetry) and implemented the ANFIS (Adaptive Neuro-Fuzzy Inference System) data assimilation (DA) to increase the spatial and temporal resolution. Although the DA results are successful, while no physical constraint is imposed. In this study, incompressible continuity equation governed mass conservation constraint is implemented by using automatic differentiation. DA is accomplished with the PINN for enhancing resolutions of the flow past the side mirror model. The increased spatial resolution of 3D velocity and vorticity fields with the trained PINN is compared with the ANFIS result. The simplified architecture of both models is shown in Figure 1.

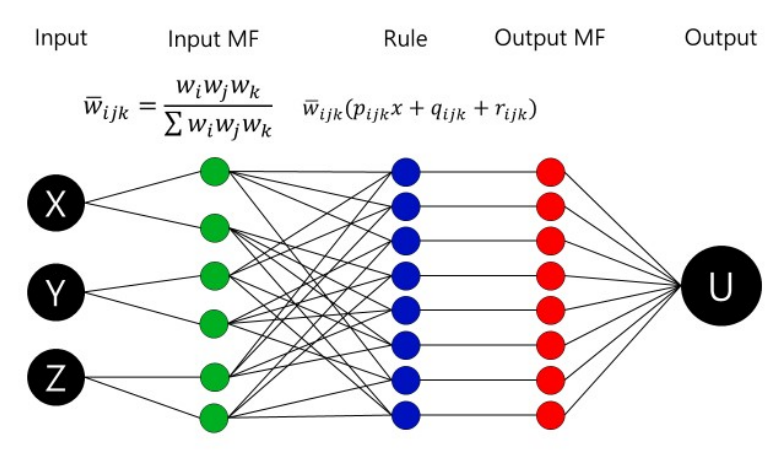

(a) ANFIS

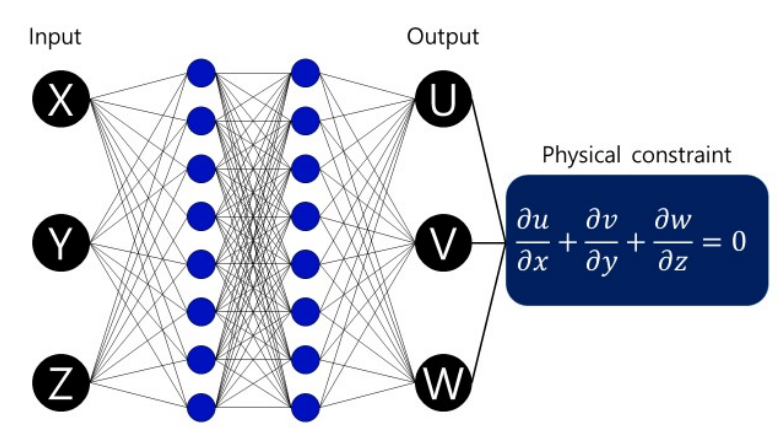

(b) PINN

Figure 1: Basic architecture of the (a) ANFIS and (b) PINN network.

As shown in Figure 2, the blunt side mirror model is placed in front of the analysis volume where marked by a red box. The flow condition is $\operatorname{Re}_{H}=85,752\left(H=0.1 \mathrm{~m}, \mathrm{v}=0.00001516 \mathrm{~m}^{2} / \mathrm{s}\right)$, upstream flow velocity is $13 \mathrm{~m} / \mathrm{s}$. The Helium Filled Soap Bubbles (HFSB) was used for tracking particles, Universal UR5 robotic arm and Quantronix Darwin-Duo Nd YLF laser (21V, 10A), four CMOS cameras were used to measure time-resolved 3D velocity fields. The frame rate was $856 \mathrm{fps}$. Detail description about experimental method and further results of the measurement are available from Kim et al. (2021). 
The input data of ANFIS is obtained at $0 ; \mathrm{x} / \mathrm{H} ; 1.5,-0.7 ; \mathrm{y} / \mathrm{H} ; 0.7,0 ; \mathrm{z} / \mathrm{H} ; 1.3$ region and nodes of that are 30, 28 and 26, respectively. Total nodes are 21,840 and the $U / U_{\infty}, V / U_{\infty}, W / U_{\infty}$ are used to labels. We shuffle all data before separate the data. $70 \%$ of inputs and labels are training data and remaining $30 \%$ are test data. Sugeno-type Fuzzy inference which suggested by Jang (1993) is implemented and 6 generalized bell functions are used to input membership function. Total train epochs are 20,000.

Same inputs and labels are applied to PINN training. The first and last layer have 3 neural and the other 10 layers have 250 neural. Total training epochs are 30,000. ANFIS and PINN are trained on a single NVIDIA RTX 3080 GPU card and it took 2 hours and 4 hours for whole training, respectively.

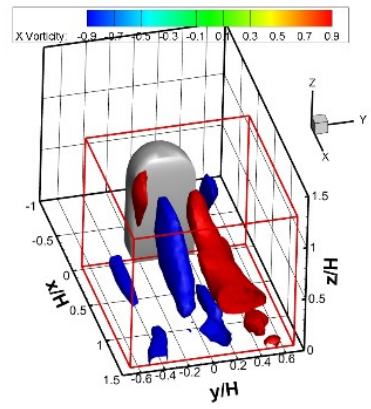

(a) ANFIS

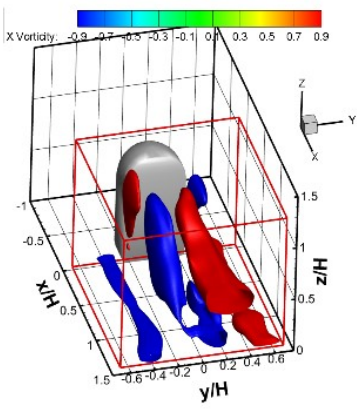

(b) PINN

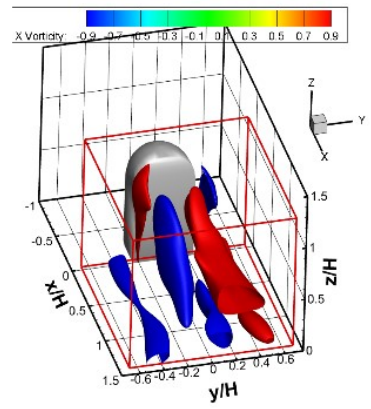

(c) PINN

Figure 2: Average streamwise vorticity by (a) raw and step size x 4 (b) ANFIS, (c) PINN

ANFIS and PINN model's test Root Mean Squared Error (RMSE) regarding to streamwise velocity are 0.013 and 0.0128 , respectively. Also, the second-order continuity equation is calculated to compare the Raw data, ANFIS and PINN on the step size x1, and the result is 1.56, 3.92 and 0.65 , respectively. This result demonstrates the role of physical constraint, which restricts the model diverge from the physics and complies with the physical law. The vortex are calculated using predicted velocity. Figure 2(b) and 2(c) show the clear vortex structures than obtained by raw data and show the connected horseshoe vortex. In addition, Figure 2(c) has more intense vortex region behind the obstacle than the others.

Comparison between PINN and ANFIS based DA is following. Both of them have just 0.013 and 0.0128 RMSE. So, they have great potential for data assimilation to increase the spatial resolution. But, PINN method has smaller error in mass conservation (continuity equation) which means the using PINN is more desirable for physical meaning informed DA. Therefore, PINN can be considered to have more remarkable potential on the data assimilation of experimental fluid mechanics.

\section{Acknowledgements}

This work was supported by the National Research Foundation of Korea (NRF) grant, which is funded by the Korean government (MSIT) (No. 2020R1A5A8018822).

\section{References}

Jang JS (1993) Anfis: adaptive-network-based fuzzy inference system. IEEE Transactions on Systems, Man, and Cybernetics 23:665-685

Kim D, Safdari A, and Kim KC (2021) Sound pressure level spectrum analysis by combination of 4d ptv and anfis method around automotive side-view mirror models. Scientific Reports 11

Raissi M, Yazdani A, and Karniadakis GE (2020) Hidden fluid mechanics: Learning velocity and pressure fields from flow visualizations. Science 367:1026-1030 